Case Report

\title{
Severe Metformin Poisoning Successfully Treated with Simultaneous Venovenous Hemofiltration and Prolonged Intermittent Hemodialysis
}

\author{
Dovile Leonaviciute $\mathbb{D I}^{1},{ }^{1}$ Bo Madsen, ${ }^{2}$ Anne Schmedes, ${ }^{3}$ \\ Niels H. Buus, ${ }^{2}$ and Bodil S. Rasmussen ${ }^{1,4}$ \\ ${ }^{1}$ Department of Anaesthesiology and Intensive Care Medicine, Aalborg University Hospital, Aalborg, Denmark \\ ${ }^{2}$ Department of Nephrology, Aalborg University Hospital, Aalborg, Denmark \\ ${ }^{3}$ Department of Biochemistry and Immunology, Lillebaelt Hospital, Vejle, Denmark \\ ${ }^{4}$ Department of Clinical Medicine, Aalborg University, Aalborg, Denmark
}

Correspondence should be addressed to Dovile Leonaviciute; dole@rn.dk

Received 2 March 2018; Accepted 3 April 2018; Published 8 May 2018

Academic Editor: Anita J. Reddy

Copyright (C) 2018 Dovile Leonaviciute et al. This is an open access article distributed under the Creative Commons Attribution License, which permits unrestricted use, distribution, and reproduction in any medium, provided the original work is properly cited.

Metformin poisoning is a life-threatening condition with a high mortality rate. We present a patient case of metformin poisoning following intake of $80 \mathrm{~g}$ metformin resulting in severe lactate acidosis with a nadir $\mathrm{pH}$ of 6.73 and circulatory collapse, successfully treated with addition of prolonged intermittent hemodialysis (HD) to continuous venovenous hemofiltration (CVVH). The patient's $\mathrm{pH}$ became normal 48 hours after metformin ingestion during simultaneous CVVH and addition of 22 hours of intermittent HD in the ICU. The highest metformin level was found to be $991 \mu \mathrm{mol} / \mathrm{L}$ (therapeutic range 3.9-23.2 $\mu \mathrm{mol} / \mathrm{L}$ ). We conclude that in cases of severe metformin poisoning with circulatory shock and extreme lactic acidosis, the usual CVVH modality might not efficiently clear metformin. Therefore, additional prolonged HD should be considered even in the state of cardiovascular collapse with vasopressor requirement.

\section{Introduction}

Metformin is widely used and is the most frequently prescribed oral antidiabetic drug of the biguanide family [1]. Metformin inhibits hepatic gluconeogenesis and glycogenolysis and enhances peripheral glucose utilisation in patients with non-insulin-dependent diabetes [2,3]. Metformin use is generally safe and well tolerated. However, lactic acidosis is a well-known complication to metformin treatment, especially in intentional overdose or in cases of renal insufficiency. Metformin poisoning is a life-threatening condition with a very high mortality rate. With the modern intensive care treatment, mortality ranges from $50 \%$ to $30 \%$ [4]. However, those intentional overdose patients with severe acidosis $(\mathrm{pH}$ less than 6.9) have a mortality rate as high as $83 \%$ [5]. We present a patient case of metformin poisoning following intake of $80 \mathrm{~g}$ metformin resulting in severe lactate acidosis with a nadir $\mathrm{pH}$ of 6.73 and circulatory collapse, successfully treated with addition of prolonged intermittent hemodialysis (HD) to continuous venovenous hemofiltration (CVVH).

\section{Case Presentation}

A 54-year-old patient with a body weight of $66 \mathrm{~kg}$ and a medical history of anxiety, type 2 diabetes, and previous stroke was admitted to the local hospital one hour after ingestion of 80 grams of metformin in a suicide attempt. At the Emergency Department, an aspiration of the gastric content was performed followed by administration of activated charcoal. The patient developed vomiting and diarrhoea. Arterial blood gas showed pH 7.33, $\mathrm{PaCO}_{2} 4.8 \mathrm{kPa}, \mathrm{PaO}_{2} 12.8 \mathrm{kPa}$, bicarbonate $19.3 \mathrm{mmol} / \mathrm{L}$, base deficit $7 \mathrm{mmol} / \mathrm{L}$, lactate $5.9 \mathrm{mmol} / \mathrm{L}$, and blood glucose $17.9 \mathrm{mmol} / \mathrm{L}$. The patient was transferred to a university hospital. Three hours after ingestion of metformin, the metabolic acidosis had deteriorated with serum lactate $8.5 \mathrm{mmol} / \mathrm{L}$ and $\mathrm{pH}$ 7.23. Urgent hemodialysis (HD) was 


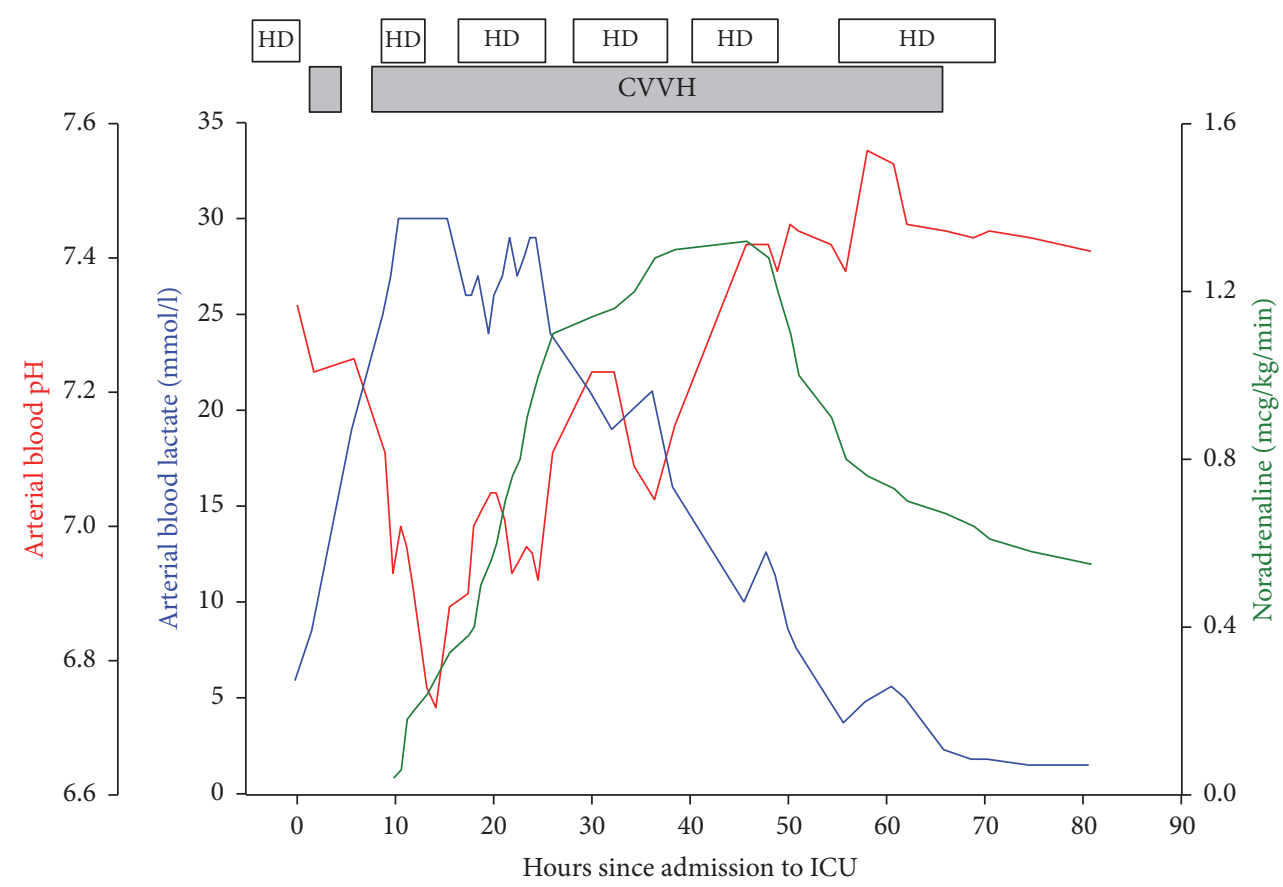

FIGURE 1: Measurements of arterial blood $\mathrm{pH}$ (red) and lactate (blue) together with the infusion rate of noradrenaline (green) in the hours following admission to the intensive care unit (ICU). HD denotes hemodialysis and CVVH continuous venovenous hemofiltration.

performed for 4 hours (h) by the nephrology department with a blood flow of $150 \mathrm{~mL} / \mathrm{min}$ and a Fresenius FX60 dialyzer (Fresenius, Bad Homburg, Germany) with a surface area of $1.4 \mathrm{~m}^{2}$. During HD, the patient became increasingly acidotic and circulatory unstable and was admitted to the intensive care unit (ICU) for circulatory support and continuous CVVH. CVVH (Aquarius, Edwards Lifesciences, Irvine, USA) was initiated with a blood flow of $300 \mathrm{~mL} / \mathrm{min}$ and a replacement fluid rate of $2000 \mathrm{~mL} / \mathrm{h}(30 \mathrm{~mL} / \mathrm{kg} / \mathrm{h})$ with $35 \mathrm{mmol} / \mathrm{L}$ bicarbonate in Accusol 35 (Baxter Healthcare Ltd., Norfolk, United Kingdom) with $50 \%$ of the replacement fluid infused prefilter and 50\% postfilter via a dialysis catheter placed in the right internal jugular vein. Dialyzer used was Aquamax HF 12 (Nikkiso, Bellco, Italy) with a surface area of $1,2 \mathrm{~m}^{2}$. Because of filter clotting, CVVH stopped after one hour. The metabolic acidosis escalated further with a $\mathrm{pH}$ decreasing to 6.73 and a serum lactate above the upper measuring limit of $30 \mathrm{mmol} / \mathrm{L}$ (ABL800 Flex, Radiometer, Copenhagen, Denmark). The patient was less conscious but managed to hyperventilate to a $\mathrm{PaCO}_{2}$ of $2.6 \mathrm{kPa}$ and base deficit of $29 \mathrm{mmol} / \mathrm{L}$. He developed severe hypotension, oliguria, hypothermia with core temperature $32.2^{\circ} \mathrm{C}$, and hypoglycaemia with a nadir blood glucose of $2.3 \mathrm{mmol} / \mathrm{L}$. Noradrenaline infusion was initiated with increasing dosage to keep mean arterial pressure above $60 \mathrm{mmHg}$. Bicarbonate $8.4 \%$ infusion was continued together with volume substitution with crystalloids. CVVH was restarted after 3 hours. Active external rewarming with forced-air warming blanket (Model 30000, 3M Health Care, St. Paul, USA) and maximal possible temperature of $39^{\circ} \mathrm{C}$ on $\mathrm{CVVH}$ was used to treat the hypothermia, but the temperature did not increase. As the patient's condition deteriorated, the hemodynamic instability progressed, cardiac arrhythmias occurred, and serum lactate remained immeasurably high. Focus Assessed Transthoracic Echocardiography (FATE) revealed a normal systolic function of both ventricles.

Because CVVH alone failed to stabilise the patient's condition and despite of hemodynamic instability and vasopressor requirement (noradrenaline infusion $0.4 \mathrm{mcg} / \mathrm{kg} / \mathrm{min}$ ), a decision was made to start HD simultaneously with CVVH. An extra dialysis catheter was placed in the left internal jugular vein and intermittent $\mathrm{HD}$ with a blood flow of $300 \mathrm{~mL} / \mathrm{min}$, Fresenius FX80 dialyzer (Fresenius, Bad Homburg, Germany) with a surface area of $1.8 \mathrm{~m}^{2}$, with the highest possible concentration of bicarbonate buffer of $40 \mathrm{mmol} / \mathrm{L}$ on $\mathrm{HD}$ machine, was simultaneously performed for $4 \mathrm{~h}$ and repeated for $8 \mathrm{~h}$ on the first day. On the second day, HD was performed twice for $8 \mathrm{~h}$ and on the third day for $16.5 \mathrm{~h}$, while CVVH was continued simultaneously for almost $58 \mathrm{~h}$. Progression of the acidosis occurred with a drop in $\mathrm{pH}$ whenever HD was paused (Figure 1).

On the second day in the ICU, the patient was intubated and mechanical ventilation was initiated, because of respiratory insufficiency due to increasing $\mathrm{PaCO}_{2}$. Only $48 \mathrm{~h}$ after metformin ingestion and $36 \mathrm{~h}$ in the ICU with cardiopulmonary support using very high doses of noradrenaline infusion (up to $1.4 \mathrm{mcg} / \mathrm{kg} / \mathrm{min}$ ) and CVVH combined with $22 \mathrm{~h}$ in total of intermittent HD with bicarbonate buffer, the patient's $\mathrm{pH}$ finally normalized. Serum lactate was still elevated but decreased very slowly to $2 \mathrm{mmol} / \mathrm{L}$ on the third day during the continued treatment with $\mathrm{CVVH}$ and intermittent $\mathrm{HD}$. After $58 \mathrm{~h}$ on both $\mathrm{CVVH}$ and intermittent $\mathrm{HD}$, the CVVH was discontinued and the patient remained on intermittent HD because of acute oliguric renal failure 
until day 15. The patient had an increase of international normalized ratio (INR) from 1.2 at the day of admission to a maximal value of 2.0 at day 2. The patient's condition improved gradually and he was discharged from the hospital after 7 weeks. At that time, the patient was still nourished via a percutaneous endoscopic gastrostomy tube due to dysphagia and used a walker due to polyneuropathy. The patient's renal function on discharge was normal.

A metformin plasma concentration, determined by high pressure liquid chromatography tandem mass spectrometry (Waters Acquity UPLC, Xevo TQ-S), was later measured from the plasma obtained $14 \mathrm{~h}$ after poisoning (after $4 \mathrm{~h}$ of $\mathrm{HD}$ and one hour of $\mathrm{CVVH}$ ) and was found to be as high as $991 \mu \mathrm{mol} / \mathrm{L}$ (therapeutic range 3.9-23.2 $\mu \mathrm{mol} / \mathrm{L}$ ). $48 \mathrm{~h}$ after poisoning, remaining on CVVH and additional 20 hours of intermittent $\mathrm{HD}$, metformin concentration was reduced to $327 \mu \mathrm{mol} / \mathrm{L}$. After additional 8 hours of HD and CVVH, metformin concentration was further reduced to $84.4 \mu \mathrm{mol} / \mathrm{L}$ at $62 \mathrm{~h}$ after poisoning.

\section{Discussion}

Severe metformin poisoning often presents with a profound lactic acidosis followed by collapse of the cardiovascular system. Symptoms of metformin poisoning are diffuse with abdominal pain, nausea, vomiting, hypothermia, decreased level of consciousness, and circulatory instability leading to multiorgan failure. The circulatory instability is due to peripheral vasoplegia as described in many case reports where low systemic vascular resistance was measured [6]. We observed a clinical picture of a hyperdynamic circulatory state where FATE revealed normal contractility of the heart together with severe systemic hypotension. Vasoplegia and vasodilatation can explain hypothermia, which is a common symptom of metformin poisoning [6].

Treatment of metformin poisoning is symptomatic and supportive and there is no antidote available. Typical treatment strategies consist of correcting acidosis with intravenous sodium bicarbonate and decreasing the blood levels of metformin. Further prevention of gastrointestinal absorption can be achieved with activated charcoal. In case of renal insufficiency, renal replacement therapy is the only option for metformin removal and acidosis correction.

Metformin is readily dialyzable. It is a small molecule with a molecular weight of $165 \mathrm{Da}$, not protein bound, and after gastrointestinal absorption it rapidly moves into the tissue compartment and has a large volume of distribution of 63-276 L (1-5 L/kg) [1]. Lalau et al. previously demonstrated a biphasic pattern of metformin elimination according to a two-compartment model. This two-compartment model suggests that a brief hemodialysis session is not sufficient in eliminating metformin due to a rebound phenomenon [7]. These pharmacokinetic properties indicate a need of prolonged dialysis for metformin elimination. Several case reports describe favourable outcome after severe metformin poisoning treated with prolonged intermittent HD. These reports suggest that prolonged $\mathrm{HD}(>15 \mathrm{~h})$ is needed and indicated in patients, who suffer from a severe overdose and who are able to tolerate $\operatorname{HD}[2,8]$. In our case, $62 \mathrm{~h}$ after ingestion and with ongoing treatment with $\mathrm{CVVH}$ and additional $32 \mathrm{~h}$ of intermittent $\mathrm{HD}$, the metformin level was still elevated to $84.4 \mu \mathrm{mol} / \mathrm{L}$, emphasizing the need for prolonged HD in severe metformin overdose.

Usually intermittent $\mathrm{HD}$ is poorly tolerated in critically ill patients with hemodynamic instability [9]. In the ICU setting CVVH or continuous venovenous hemodiafiltration (CVVHDF) is used because fluid shifts are less profound and therefore applicable in hemodynamic unstable patients. However, these modalities need high ultrafiltration rates for successful treatment in case of metformin intoxication [10], but clearance of metformin by continuous venovenous treatment modalities is still 3-4 times less than clearance by conventional HD $[1,11]$. Panzer reported successful use of two CVVH machines simultaneously for hemodynamically unstable patient to facilitate high volume CVVH [12]. Friesecke has successfully applied the same approach with two dialysis catheters and used discontinuous hemofiltration (highflux) in addition to CVVH for severe circulatory unstable patient. To our knowledge, the use of intermittent HD in a patient with metformin poisoning with severe hemodynamic instability and such dependence on vasopressors is described only once [13]. However, the placement of two dialysis catheters in the jugular vein position may theoretically lead to a recirculation between them, if the tips of the catheters are placed close to each other. The jugular and femoral placement of the catheters will eliminate this possibility.

Substitution with bicarbonate in case of lactic acidosis is controversial as there are concerns that treatment with bicarbonate can increase intracellular acidosis [14]. However, bicarbonate treatment is widely used to stabilise metabolic acidosis in cases of metformin poisoning as described in the literature. We used high doses of bicarbonate as intravenous infusion and highest possible concentration of bicarbonate buffer in the HD machine simultaneously with CVVH with $35 \mathrm{mmol} / \mathrm{L}$ bicarbonate in the Accusol 35 (Baxter) replacement fluid. We observed worsening of the acidosis and cardiovascular status when HD was paused and we presume this deterioration to be due to lack of bicarbonate buffer. Renal replacement therapy, including conventional HD and CVVH, offers both theoretical and practical advantages over bicarbonate infusion. These allow for isovolemic correction of the metabolic acidosis while removing metformin and lactate [6].

Recent recommendations for metformin poisoning from the Extracorporeal Treatments in Poisoning Workgroup advocate for intermittent $\mathrm{HD}$, but continuous renal replacement therapies may be considered if $\mathrm{HD}$ is unavailable [1]. Initiation of dialysis is suggested in very severe cases, when lactate concentration is $>15 \mathrm{mmol} / \mathrm{L}$ and $\mathrm{pH}<7.0$ together with shock or organ failure [1]. In our opinion, these recommendations are more applicable in situations of metformin-associated lactic acidosis, as in acute high dose metformin poisoning the acidosis accelerates very fast and the patient's condition deteriorates dramatically with development of circulatory collapse.

We therefore suggest that in cases of acute high dose metformin poisoning HD should be initiated earlier than that stated in the recommendations. 


\section{Conclusion}

In cases of severe metformin poisoning with shock and extreme lactic acidosis usual CVVH modality might not effectively clear metformin and additional prolonged intermittent HD should be considered instead or together with CVVH, even in the state of cardiovascular collapse with vasopressor requirement.

\section{Conflicts of Interest}

The authors declare no conflicts of interest regarding the publication of this article.

\section{References}

[1] D. P. Calello, K. D. Liu, T. J. Wiegand et al., "Extracorporeal treatment for metformin poisoning: Systematic review and recommendations from the extracorporeal treatments in poisoning workgroup," Critical Care Medicine, vol. 43, no. 8, pp. 1716-1730, 2015.

[2] P. Y. F. Guo, L. J. Storsley, and S. N. Finkle, "Severe lactic acidosis treated with prolonged hemodialysis: Recovery after massive overdoses of metformin," Seminars in Dialysis, vol. 19, no. 1, pp. 80-83, 2006.

[3] C. J. Bailey and R. C. Turner, "Metformin," The New England Journal of Medicine, vol. 334, no. 9, pp. 574-579, 1996.

[4] N. Peters, N. Jay, and D. Barraud, "Metformin-associated lactic acidosis in an intensive care unit," Critical Care, vol. 12, no. 6, article R149, 2008.

[5] D. M. Dell'Aglio, L. J. Perino, Z. Kazzi, J. Abramson, M. D. Schwartz, and B. W. Morgan, "Acute metformin overdose: examining serum $\mathrm{pH}$, lactate level, and metformin concentrations in survivors versus nonsurvivors: a systematic review of the literature," Annals of Emergency Medicine, vol. 54, no. 6, pp. 818-823, 2009.

[6] K. F. H. Teale, A. Devine, H. Stewart, and N. J. H. Harper, "The management of metformin overdose," Anaesthesia, vol. 53, no. 7, pp. 698-701, 1998.

[7] J. D. Lalau, M. Andrejak, P. Moriniere, and et al., "Hemodialysis in the treatment of lactic acidosis in diabetics treated by metformin: a study of metformin elimination," International Journal of Clinical Pharmacology, Therapy, and Toxicology, vol. 27, pp. 285-288, 1989.

[8] A. Seidowsky, S. Nseir, N. Houdret, and F. Fourrier, "Metformin-associated lactic acidosis: A prognostic and therapeutic study," Critical Care Medicine, vol. 37, no. 7, pp. 2191-2196, 2009.

[9] L. Brochard, F. Abroug, M. Brenner et al., "An official ATS/ERS/ ESICM/SCCM/SRLF statement: prevention and management of acute renal failure in the ICU patient: an international consensus conference in intensive care medicine," American Journal of Respiratory and Critical Care Medicine, vol. 181, no. 10, pp. 1128-1155, 2010.

[10] B. Harvey, C. Hickman, G. Hinson, T. Ralph, and A. Mayer, "Severe lactic acidosis complicating metformin overdose successfully treated with high-volume venovenous hemofiltration and aggressive alkalinization," Pediatric Critical Care Medicine, vol. 6, no. 5, pp. 598-601, 2005.

[11] F. Barrueto, W. J. Meggs, and M. J. Barchman, "Clearance of metformin by hemofiltration in overdose," Journal of Toxicology - Clinical Toxicology, vol. 40, no. 2, pp. 177-180, 2002.
[12] U. Panzer, S. Kluge, G. Kreymann, and G. Wolf, "Combination of intermittent haemodialysis and high-volume continuous haemofiltration for the treatment of severe metformin-induced lactic acidosis [5]," Nephrology Dialysis Transplantation, vol. 19, no. 8, pp. 2157-2158, 2004.

[13] S. Friesecke, P. Abel, M. Kraft, A. Gerner, and S. Runge, "Combined renal replacement therapy for severe metformin-induced lactic acidosis [18]," Nephrology Dialysis Transplantation, vol. 21, no. 7, pp. 2038-2039, 2006.

[14] S. Sabatini and N. A. Kurtzman, "Bicarbonate therapy in severe metabolic acidosis," Journal of the American Society of Nephrology, vol. 20, no. 4, pp. 692-695, 2009. 


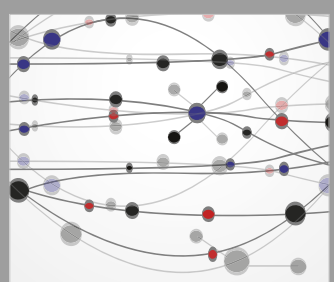

The Scientific World Journal
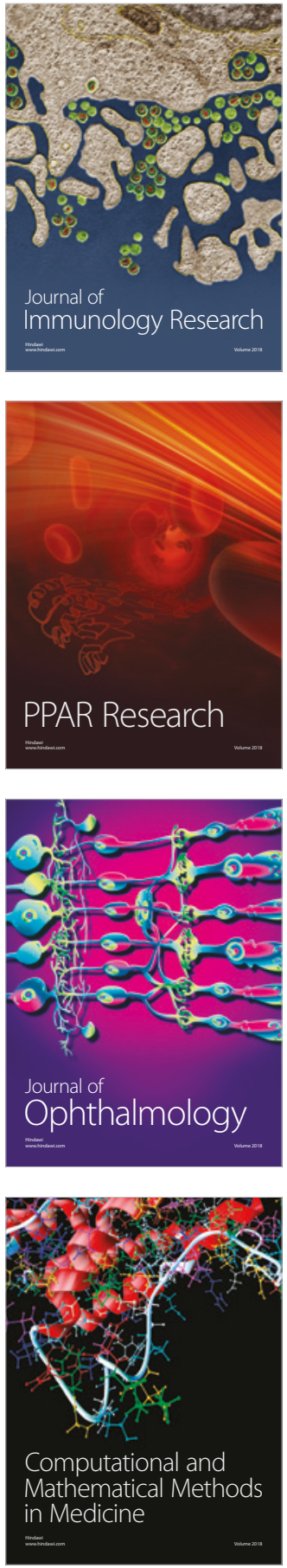

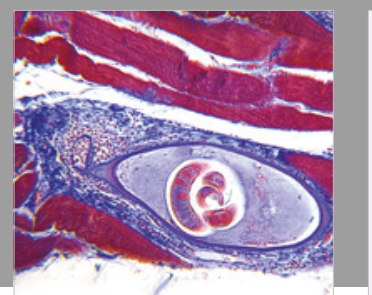

Gastroenterology Research and Practice

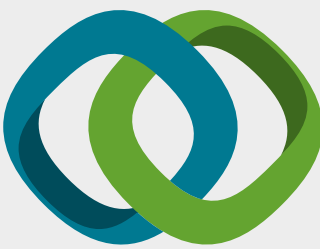

\section{Hindawi}

Submit your manuscripts at

www.hindawi.com
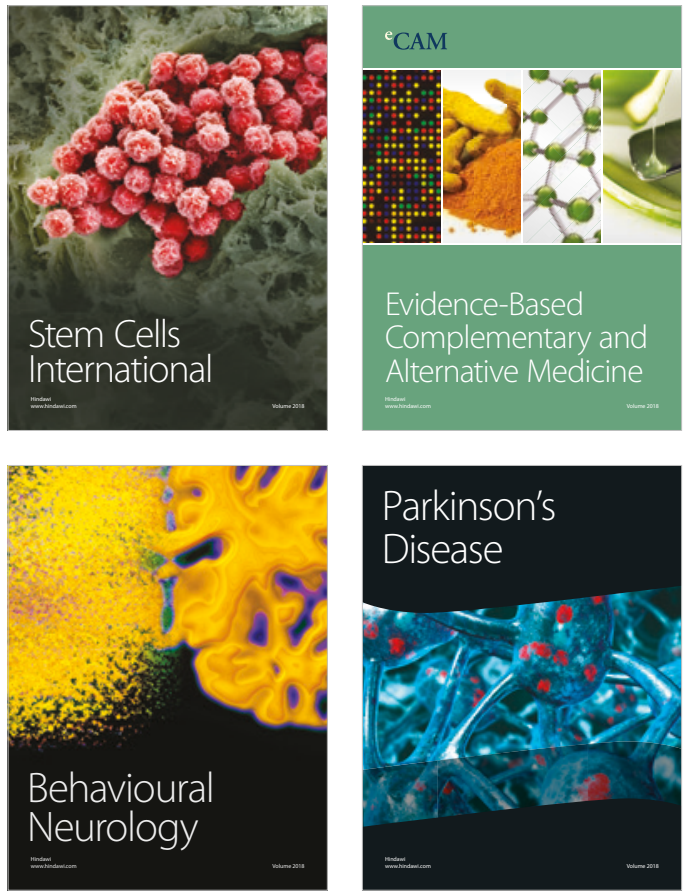

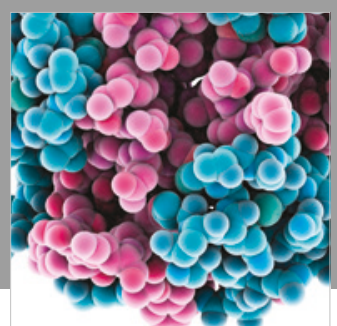

ournal of

Diabetes Research

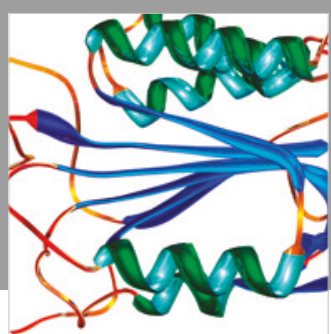

Disease Markers
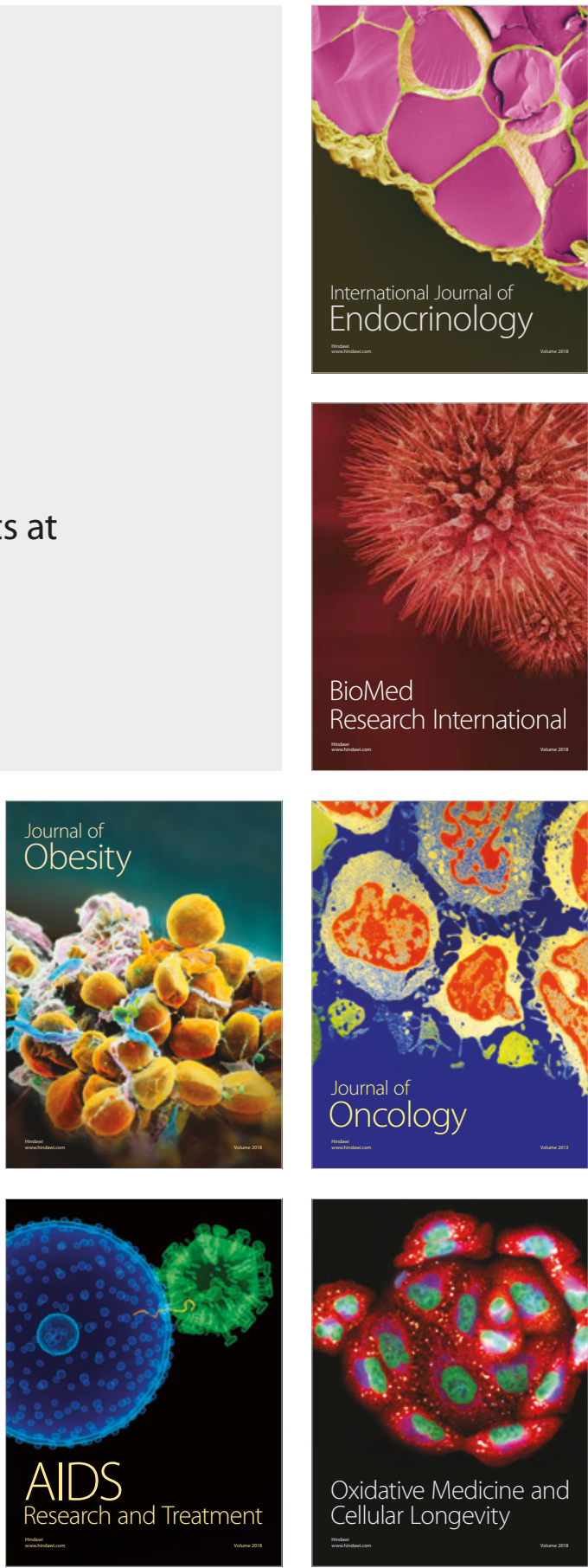